\title{
Functioning free myocutaneous anterolateral thigh flap for simultaneous reconstruction $\&$ facial reanimation in buccal carcinoma defect
}

\author{
Ravikiran Naalla, Rajan Arora, Kripa Shanker Mishra \\ *Division of Reconstructive Microsurgery, Department of Surgical Oncology, \\ Rajiv Gandhi Cancer Institute \& Research Center, New Delhi
}

DOI: 10.29322/IJSRP.11.07.2021.p11544

http://dx.doi.org/10.29322/IJSRP.11.07.2021.p11544

\begin{abstract}
Following surgical extirpation of the buccal carcinoma, there is an anatomical and functional deficit. Though the anatomical defect reconstruction is straight forward with multiple flap options, the functional deficits are addressed less frequently. Due to the resection of the facial muscles and the facial nerve branches the patients generally have facial paralysis. This facial paralysis leads to significant psychosocial consequences. We aim to present the technique of functioning free myocutaneous anterolateral thigh flap for simultaneous reconstruction \& facial reanimation.
\end{abstract}

Index Terms- Facial reanimation; anterolateral thigh flap; functioning free muscle transfer; buccal cancer

\section{INTRODUCTION}

Wide local excision (WLE) of the buccal mucosa cancers leads to resection of the facial muscles and facial nerve branches in addition to the buccal mucosa and other cheek structures. The resultant buccal mucosa defects can be resurfaced with either loco-regional flaps or free flaps. However, there has not been much focus on the treatment of facial paralysis following resection of buccal carcinoma. We aim to share our experience with anterolateral thigh (ALT) flap for simultaneous buccal mucosa defect reconstruction \& facial reanimation.

\section{CASE REPORT:}

A 40-year-old male patient was diagnosed to have a left buccal mucosa cancer (T3NOM0). He underwent a WLE of the buccal mucosa cancer, marginal mandibulectomy, upper alveolectomy and left selective neck dissection (I-IV). The resection involved the facial muscles of expression and facial nerve branches. Following resection, a myocutaneous ALT flap was harvested. The skin paddle was harvested conventionally, the vastus lateralis (VL) muscle needs to be harvested in a chimeric fashion preserving its nerve supply. The distal portion of the VL is preferred due to the presence of a fascia which is essential to provide a secure anchorage. The length of the harvested muscle depends on the distance between the anchoring points. Following the skin paddle inset, vessel anastomosis was done. The VL fascia was split into two and sutured to the orbicularis oris (distal anchor point) with 3-0 polyester suture. Along the vector of the smile, the VL was placed and anchored to the zygoma (proximal anchor point) by placing drill holes. The nerve to VL was coapted to the buccal branch of the facial nerve. This nerve can be easily identified in the parotid gland substance along with the parotid duct. Postoperative stay was uneventful. Patient underwent adjuvant radiotherapy (60 Gy). Patient gained satisfactory smile and oral sphincter competence within the five months (Figure 1).

\section{DISCUSSION:}

Buccal carcinoma resection defects can lead to debilitating functional \& psychosocial sequelae despite reconstruction with a free flap. Resection of facial muscles results in loss of facial expression and at times may lead to oral incompetence. The gracilis myocutaneous flap has been used successfully for coverage of the buccal carcinoma defect and simultaneous facial reanimation. ${ }^{1}$ However, this flap is not widely used due to the concern about harvesting a reliable skin paddle. ${ }^{2}$ To the best of our knowledge, the chimeric ALT and neurotised vastus lateralis (VL) transfer has never been reported for the reconstruction of an isolated buccal carcinoma defect. Though there might be a concern about nerve regeneration following adjuvant radiotherapy, the patient had a satisfactory nerve regeneration and outcome. Lin $\mathrm{CH}$ et al had also noted satisfactory results following adjuvant radiotherapy. 
ISSN 2250-3153

The addition of a neurotized vastus lateralis muscle not only provides facial reanimation but also preserves oral competence. The muscle also fills the dead space following resection of the soft tissue in the cheek. As the VL is bulky, this technique is best suited for patients with a large soft-tissue defects in the cheek. However, with more experience we can attempt to debulk the VL to decrease the bulk in a case-to-case basis.

\section{CONCLUSION:}

Functioning free myocutaneous anterolateral thigh flap for simultaneous reconstruction \& facial reanimation in buccal carcinoma defect is an important option in a reconstructive surgeon's armamentarium. Further studies with a larger sample size are necessary to explore the advantages of this flap application.

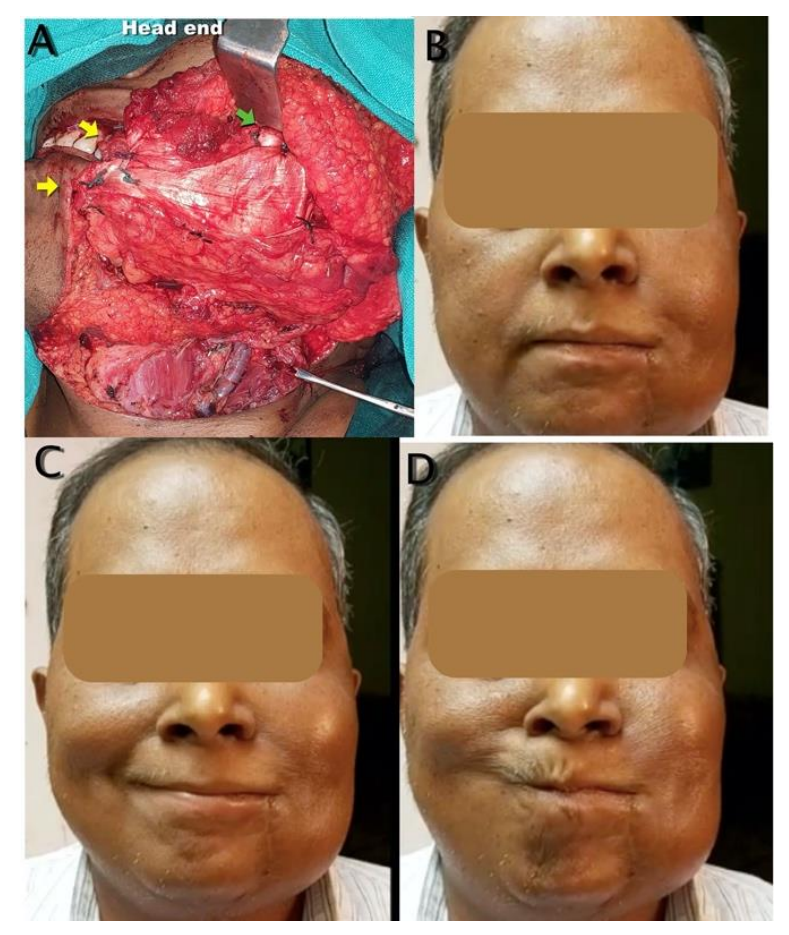

Figure 1: Image showing (A) Inset of Vastus lateralis muscle flap (green arrow- proximal anchoring point at zygoma; yellow arrowsdistal anchoring points at the upper and lower lips), (B) patient at rest, (C) attempting smile, (D) oral competence.

\section{REFERENCES}

1. Lin $\mathrm{CH}$, Wallace $\mathrm{C}$, Liao CT. Functioning free gracilis myocutaneous flap transfer provides a reliable single-stage facial reconstruction and reanimation following tumor ablation. Plast Reconstr Surg. 2011 Sep;128(3):687-696.

2. Balakrishnan TM, Jayachandiran AP, Hariraj DD, et al. Chimeric anterolateral thigh flap for primary functional reconstruction of complex, composite neartotal hemi facial post excision defects. J Craniofac Surg. 2020 Jun;31(4):e337-e343.

\section{AUTHORS}

First Author - Ravikiran Naalla, M.Ch. Consultant, Division of Reconstructive Microsurgery, Department of Surgical Oncology, Rajiv Gandhi Cancer Institute \& Research Center, New Delhi. Email: ravi_2488@yahoo.co.in

Second Author - Rajan Arora, M.Ch. Senior Consultant, Department of Surgical Oncology, Rajiv Gandhi Cancer Institute \& Research Center, New Delhi. Email: rajan_ritu@ rediffmail.com

Third Author - Kripa Shanker Mishra, M.Ch. Consultant, Division of Reconstructive Microsurgery, Department of Surgical Oncology, Rajiv Gandhi Cancer Institute \& Research Center, New Delhi. Email: drksmishra25@ gmail.com 
Correspondence Author - Ravikiran Naalla, M.Ch. Consultant, Division of Reconstructive Microsurgery, Department of Surgical Oncology, Rajiv Gandhi Cancer Institute \& Research Center, New Delhi. Email: ravi_2488@yahoo.co.in , Mobile- +919821826580 